\title{
Morphology-Dependent Reactivity of a Plasmonic Photocatalyst
}

Lin Yuan ${ }^{1,4, £}$, Minhan Lou ${ }^{2,4, £}$, Benjamin D. Clark ${ }^{1,4, £, ~ M i n g h e ~ L o u ~}{ }^{1,4}$, Linan Zhou ${ }^{1,4}$, Shu Tian $^{1,4}$, Christian R. Jacobson ${ }^{1,4}$, Peter Nordlander 2, 3, 4, * and Naomi J. Halas 1, 2, 3, 4, *

${ }^{1}$ Department of Chemistry, Rice University, Houston, Texas 77005, United States

${ }^{2}$ Department of Electrical and Computer Engineering, Rice University, Houston, Texas 77005, United States

${ }^{3}$ Department of Physics \& Astronomy, Rice University, Houston, Texas 77005, United States

${ }^{4}$ Laboratory for Nanophotonics, Rice University, Houston, Texas 77005, United States

$£$ L. Yuan, M. Lou and B. D. Clark contributed equally to this work

* corresponding authors:

halas@rice.edu;

nordland@rice.edu

Supporting information

External quantum efficiency (EQE) and surface area calculation:

The absolute HD rate ( $\mathrm{r}_{\mathrm{HD}}$ ) was converted from mass spectrometer HD intensity through calibration as reported before. ${ }^{1}$ For quantum efficiency, the formula is listed as below:

$$
E Q E=\frac{r_{H D} \times N_{A}}{P_{w} / h v} \times 100 \%
$$

Where $\mathrm{r}_{\mathrm{HD}}, \mathrm{N}_{\mathrm{A}}, \mathrm{P}_{\mathrm{w}}, \mathrm{h}$ and $\mathrm{v}$ represent the HD rate, Avogadro constant, power of incident light, planck constant and the frequency of incident light, respectively.

The surface to volume ratio (Table S1) were calculated from the statistical results of size distribution from each shape of nanoparticles, as:

$$
\sigma_{\text {surface to volume ratio }}=\frac{S_{\text {total }}}{V_{\text {total }}}
$$

And the formula for the calculation of nanocrystals, nanocubes and octopods are counted as spheres, cubes and cubes with the regular tetrahedron for 8 corners. The formulas are shown as follow:

$$
\begin{aligned}
S_{\text {nanocrystal }} & =4 \pi r^{2} \\
V_{\text {nanocrystal }} & =\frac{4}{3} \pi r^{3} \\
S_{\text {nanocube }} & =6 a^{2}
\end{aligned}
$$




$$
\begin{gathered}
V_{\text {nanocube }}=a^{3} \\
S_{\text {octopod }}=\frac{16(1+\sqrt{3})}{27} a^{2} \approx 4.61 a^{2} \\
V_{\text {octopod }}=\frac{63}{128} a^{3} \approx 0.4922 a^{3}
\end{gathered}
$$

Where $\mathrm{r}$ represents radius of sphere, and $a$ represents edge length of cube and octopod (length of two vertexes of tetrahedrons).

\section{Theoretical interband-transition contribution to the Al permittivity:}

In the Lorentz-Drude model of metals, the Al optical constant can be fitted by sum of multiple interband transitions Lorentz functions and Drude functions. Theoretical Al optical constant fitted by Rakic ${ }^{2}$ were used to approximate the interband contribution $\alpha_{\text {interband }}$ to the imaginary part of the $\mathrm{Al}$ permittivity.

$$
\alpha_{\text {interband }}=\frac{\operatorname{Im}\left(\varepsilon_{\text {Rakik,interband }}\right)}{\operatorname{Im}\left(\varepsilon_{\text {Rakic }}\right)}
$$

Figure S12 shows, between 350 and $800 \mathrm{~nm}$, interband transitions account for more than $95 \%$ of the transitions contributing to the Rakic Al permittivity. ${ }^{2}$ We assumed Palik Al dielectric constant data $^{3}$ has the same interband transitions contribution as the Rakic data. 

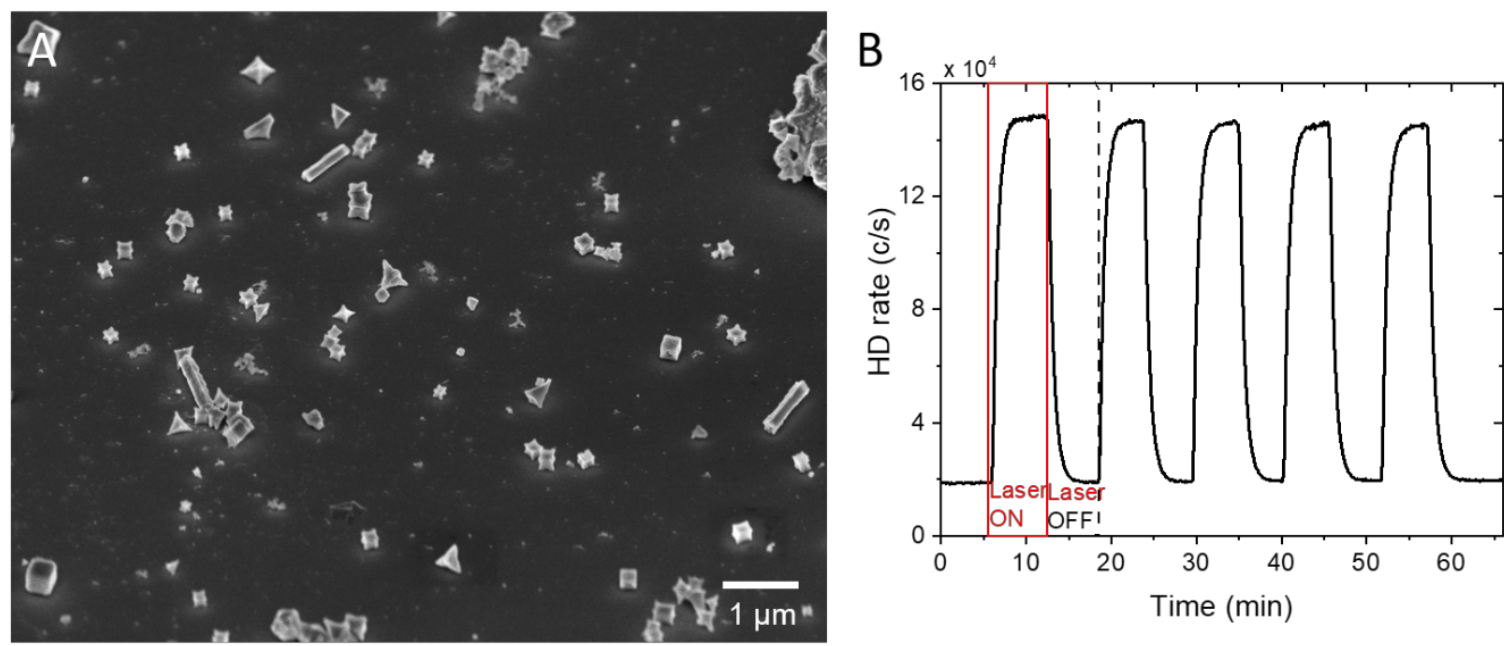

Figure S1. Al octopods solution SEM image and photocatalysis stability (A) A $45^{\circ}$ SEM image of the samples from Al octopod solution on a larger area. (B) Photocatalysis stability of $5 \% \mathrm{Al}$ octopods with the support of $\gamma-\mathrm{Al}_{2} \mathrm{O}_{3}$ under the white light irradiation at $40 \mathrm{~W} / \mathrm{cm}^{2}$, with $15 \mathrm{sccm} \mathrm{H}_{2}$ and $15 \mathrm{sccm} \mathrm{D}_{2}$ flowing.

A

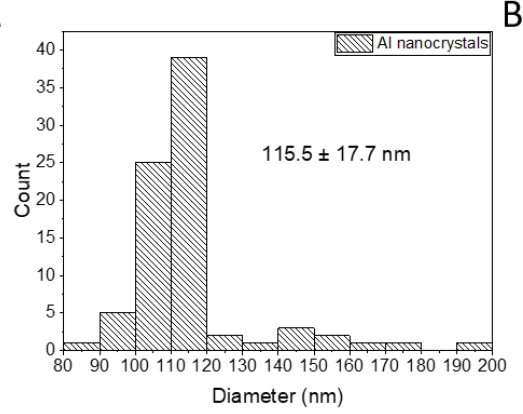

B

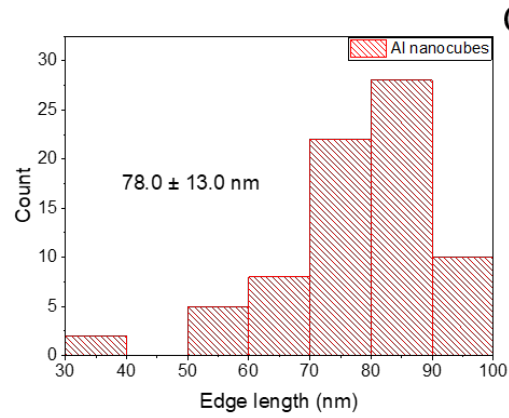

C

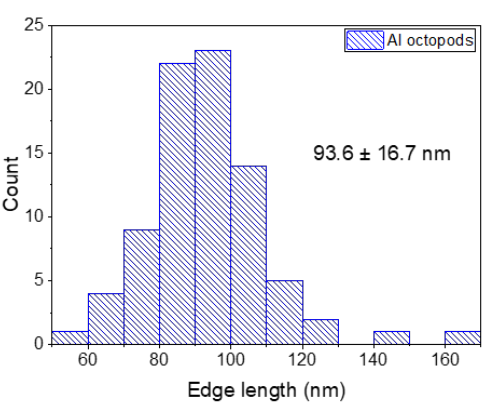

Figure S2. Size distribution of Al nanocrystals, nanocubes and octopods. (A) Diameter of A1 nanocrystals and edge length of (B) Al nanocubes and (C) octopods. 


\begin{tabular}{|c|c|c|c|}
\hline & Al nanocrystals & Al nanocubes & Al octopods \\
\hline $\begin{array}{c}\text { Surface to volume } \\
\text { ratio }\end{array}$ & 0.0493 & 0.0733 & 0.0866 \\
\hline
\end{tabular}

Table S1. Calculated surface to volume ratio of Al nanocrystals, nanocubes and octopods.
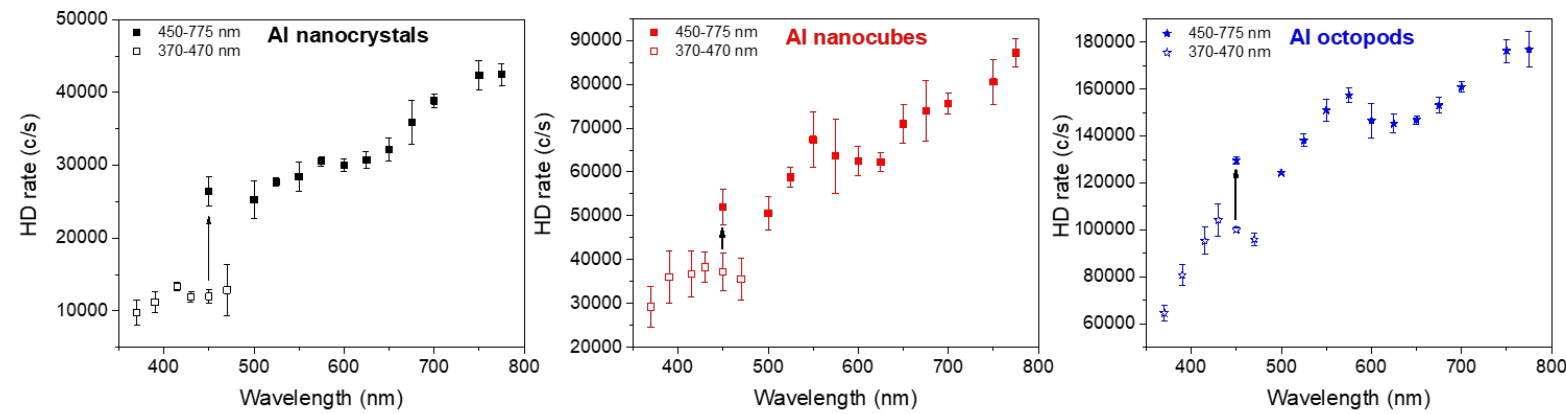

Figure S3. Wavelength dependent photocatalysis on aluminum nanoparticles using different laser (Chameleon for $370-470 \mathrm{~nm}$ and Fianium for $450-800 \mathrm{~nm}$ ). The chamber was heated at $500{ }^{\circ} \mathrm{C}$ and irradiated at $7 \mathrm{~W} / \mathrm{cm}^{2}$ at each wavelength. Frequencies of the Fianium and chameleon equipped with the harmonic box are different, so the photocatalytic rate under the similar power density varies. The only wavelength that they have overlap is at $450 \mathrm{~nm}$. The final wavelength dependent photocatalysis from $370 \mathrm{~nm}-800 \mathrm{~nm}$ was evaluated by multiplying reaction rate generated by chameleon with the times that the mean reaction rate at $450 \mathrm{~nm}$ overlaps with each other. 

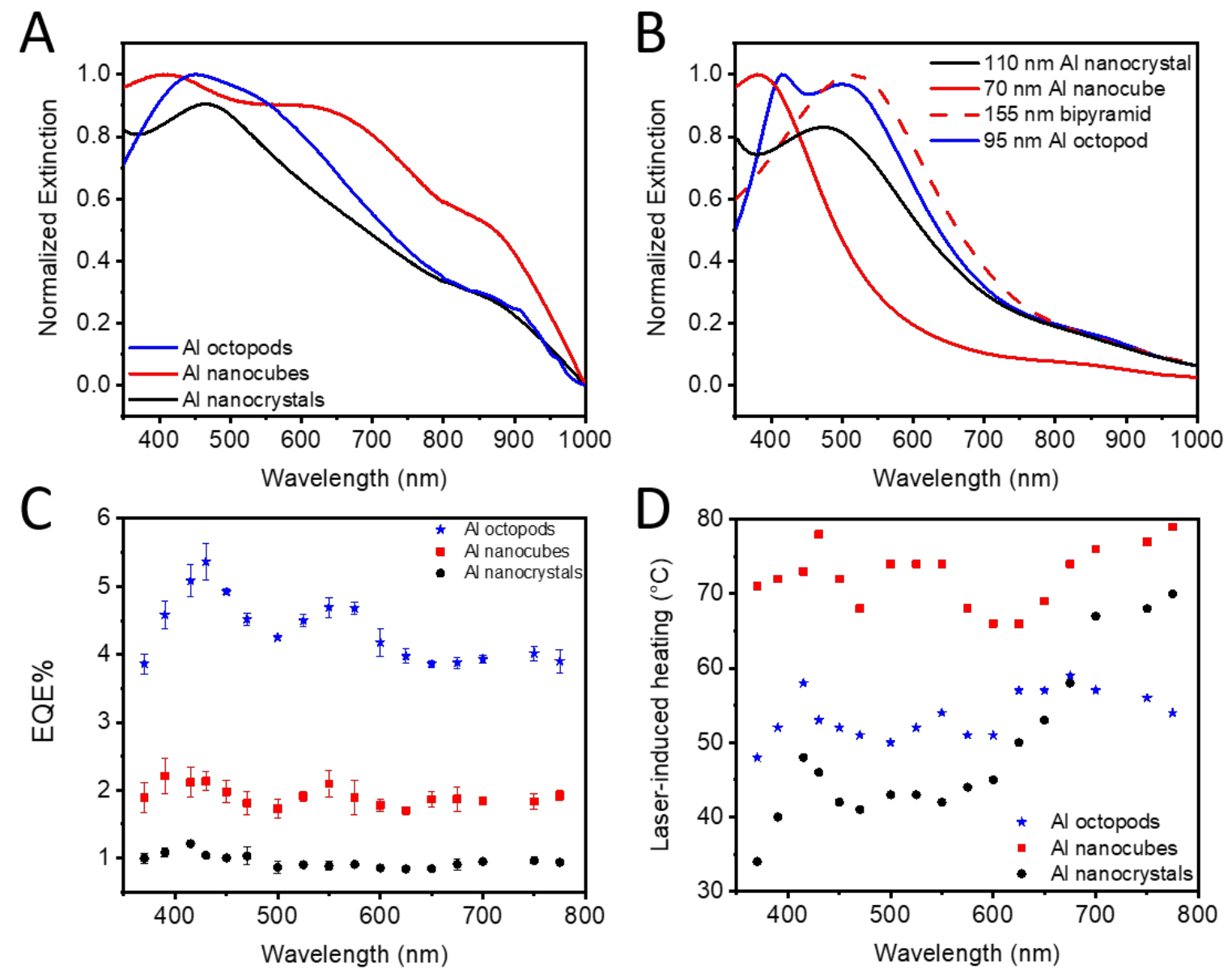

Figure S4. Extinction spectrum, wavelength dependent photocatalytic $\mathrm{H}_{2}$ dissociation, and laser-induced heating. (A) Extinction spectrum of Al nanocrystals, nanocubes, and octopods. (B) Finite-difference time-domain (FDTD) simulation of the extinction spectrum of Al nanocrystal, nanocube and octopod. (C) Wavelength dependent external quantum efficiency (EQE) on Al nanocrystals, nanocubes and octopods. (D) Laser-induced surface maximum temperature increase on the Al nanoparticles with different shapes. Error bars for (C) and (D): standard deviation of 3 independent experiments. Error bars for (D) are smaller than $2^{\circ} \mathrm{C}$. 

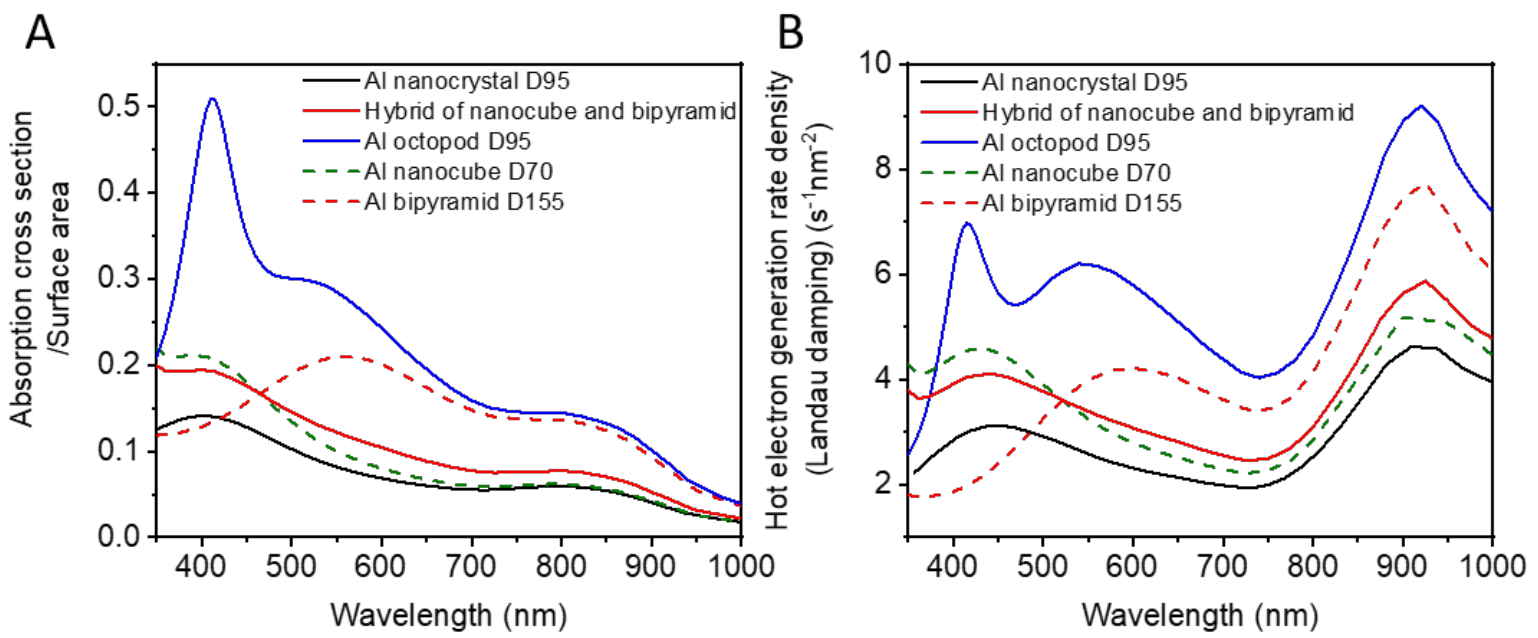

Figure S5. (A) Absorption cross section divided by nanoparticle surface area and (B) hot electrons injection rate per surface area through landau damping under incident light intensity of $1 \mathrm{~W} / \mathrm{cm}^{2}$ and adsorbate resonance linewidth $0.1 \mathrm{eV}$.

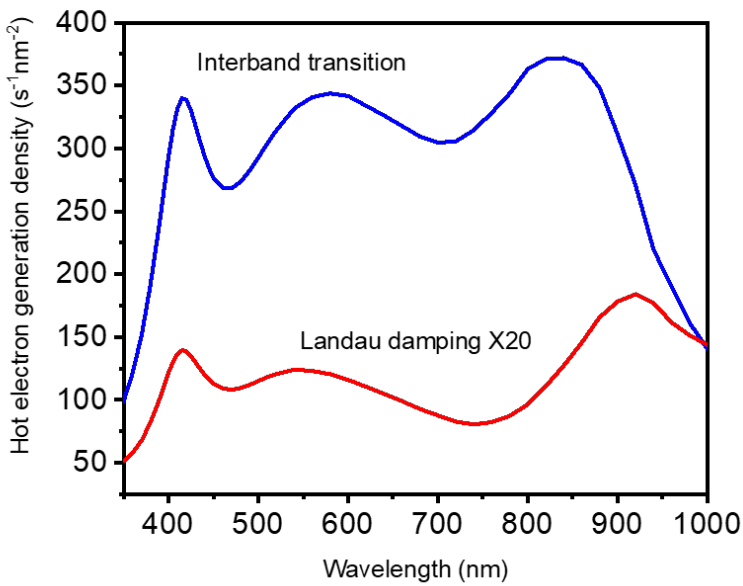

Figure S6. Comparison of wavelength dependent hot-electron injection rate per surface area through interband transition and landau damping on Al octopod under incident light intensity of $1 \mathrm{~W} / \mathrm{cm}^{2}$ and adsorbate resonance linewidth $0.1 \mathrm{eV}$. 


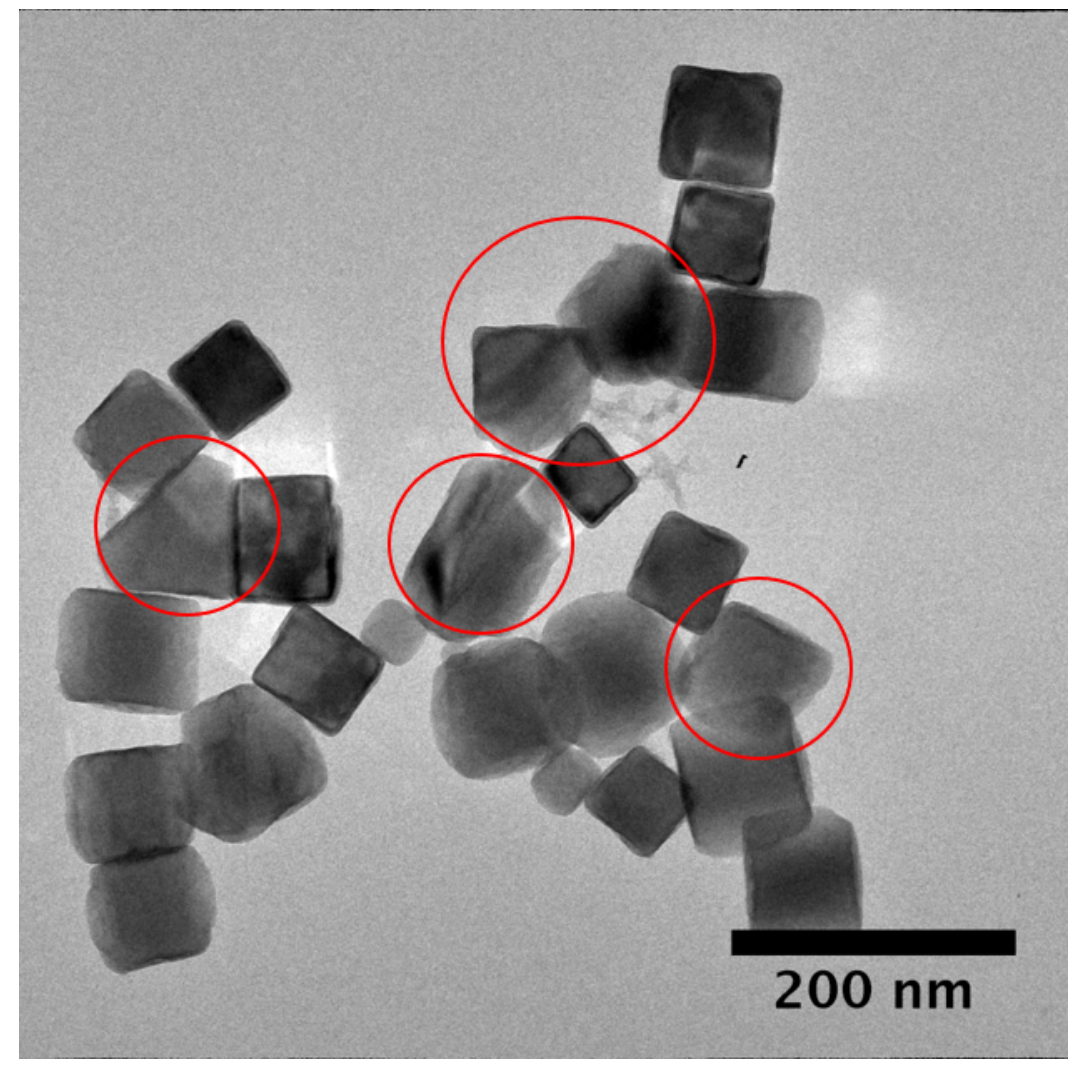

Figure S7. TEM images of Al nanocubes. Red circles show the particle with other geometries among nanocubes, such as right bipyramids and pentagonal rods. 

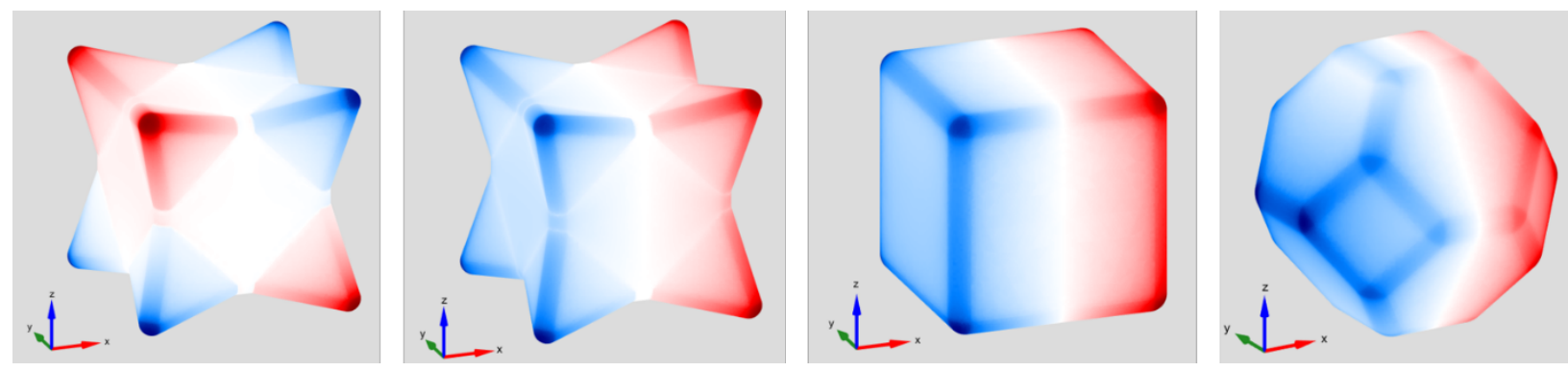

Figure S8. Charge plot of quadrupole $(420 \mathrm{~nm})$ and dipole mode $(550 \mathrm{~nm})$ of Al octopod, dipole mode $(420 \mathrm{~nm})$ of $\mathrm{Al}$ nanocube and nanocrystal, from left to right respectively.

A

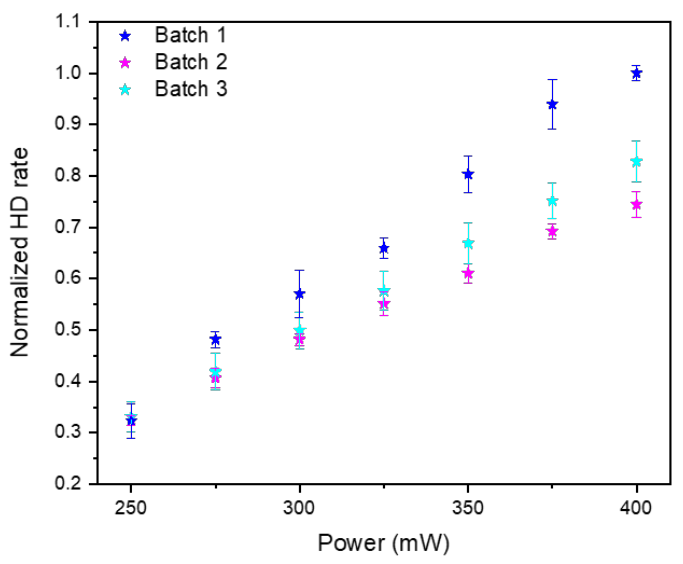

B

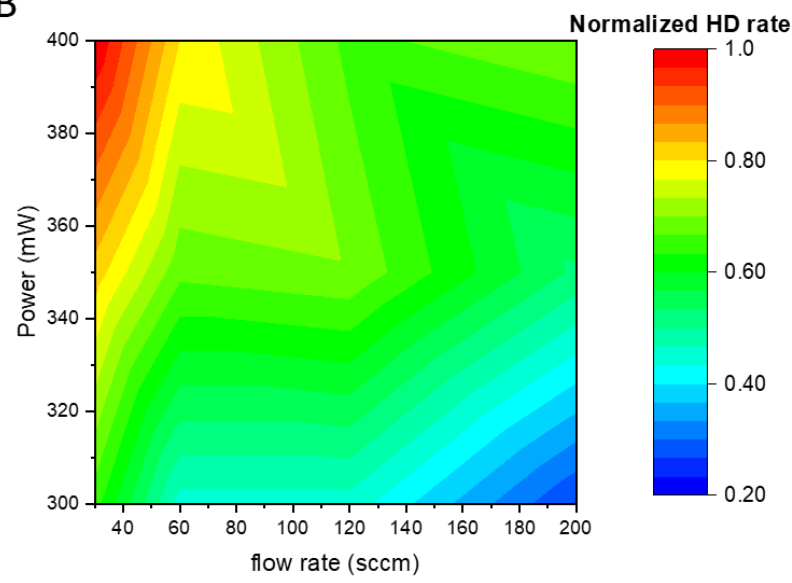

Figure S9. (A) Batch to batch differences of photocatalytic performance on Al octopods and (B) flow rate dependent experiment on Al octopods. 


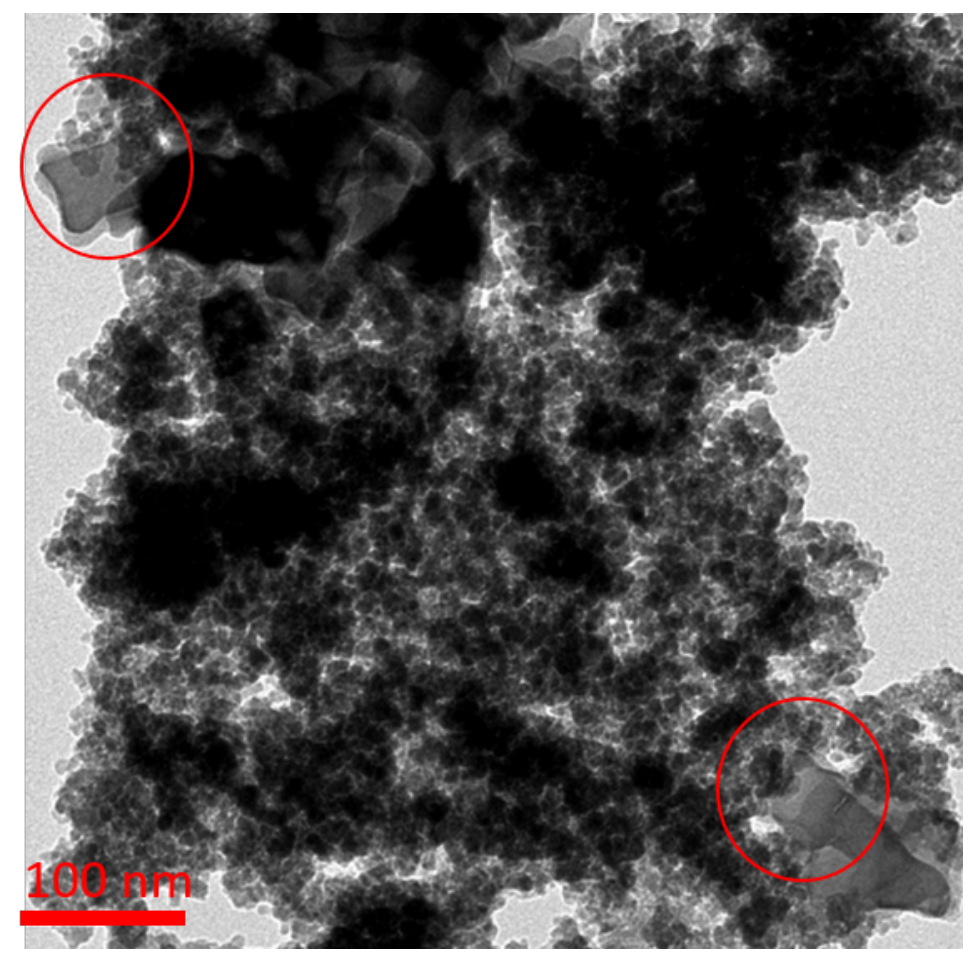

Figure S10. TEM image of $\mathrm{Al}$ octopods on $\gamma-\mathrm{Al}_{2} \mathrm{O}_{3}$ after the irradiation for 2 hrs under 40 $\mathrm{W} / \mathrm{cm}^{2}$. 


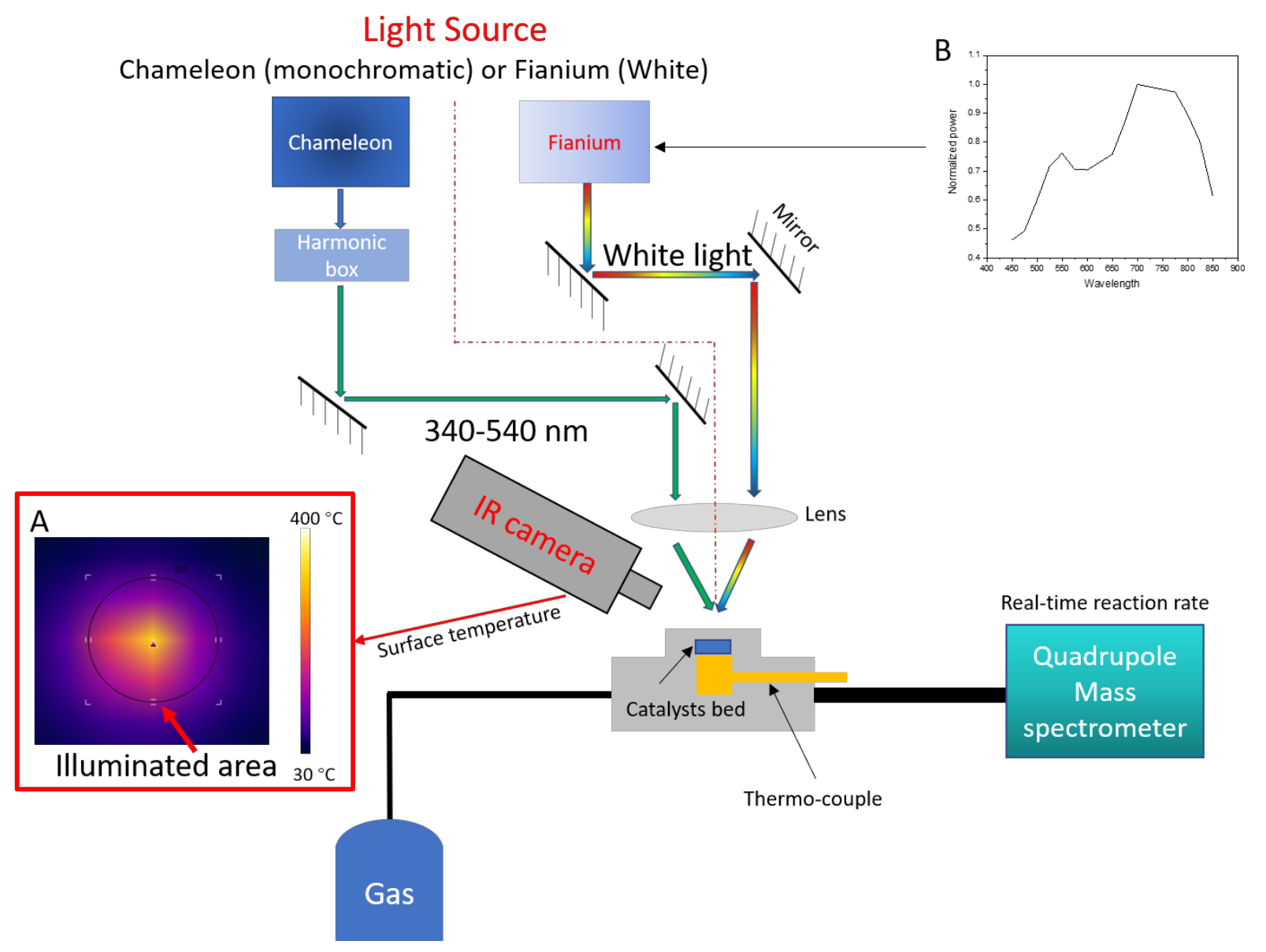

Figure S11. Schematic of the setup for photocatalysis measurement. (A) Real-time image of the temperature of the illuminated area of catalysts bed through IR camera. (B) Spectrum of the white light laser (Fianium). 


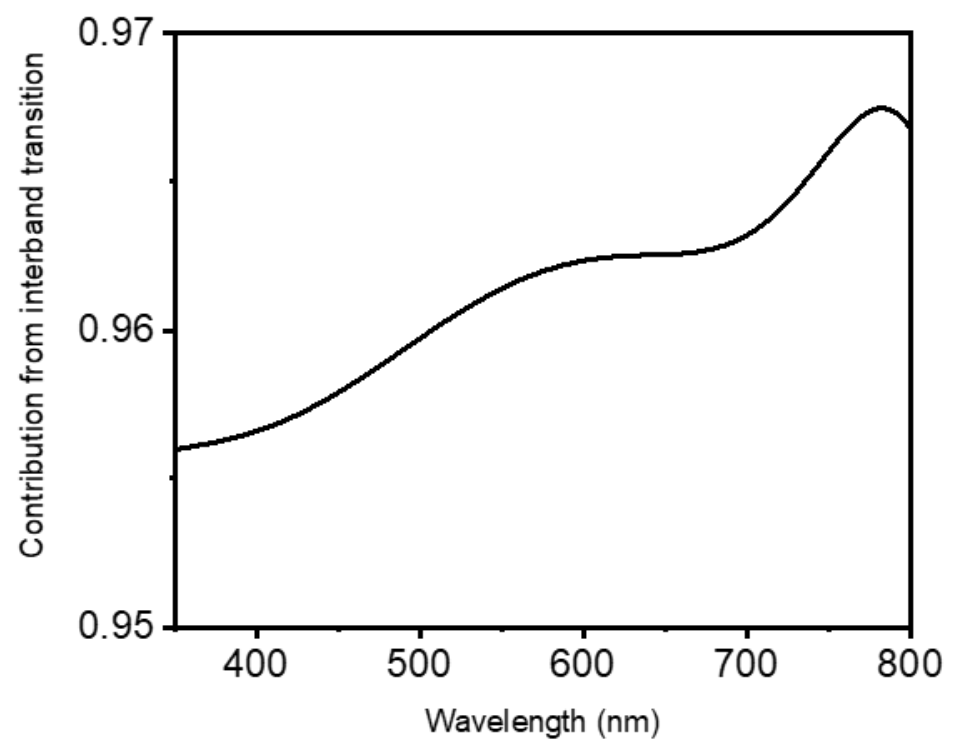

Figure S12. The interband transitions contribution to the imaginary part of Rakic Al dielectric permittivity.

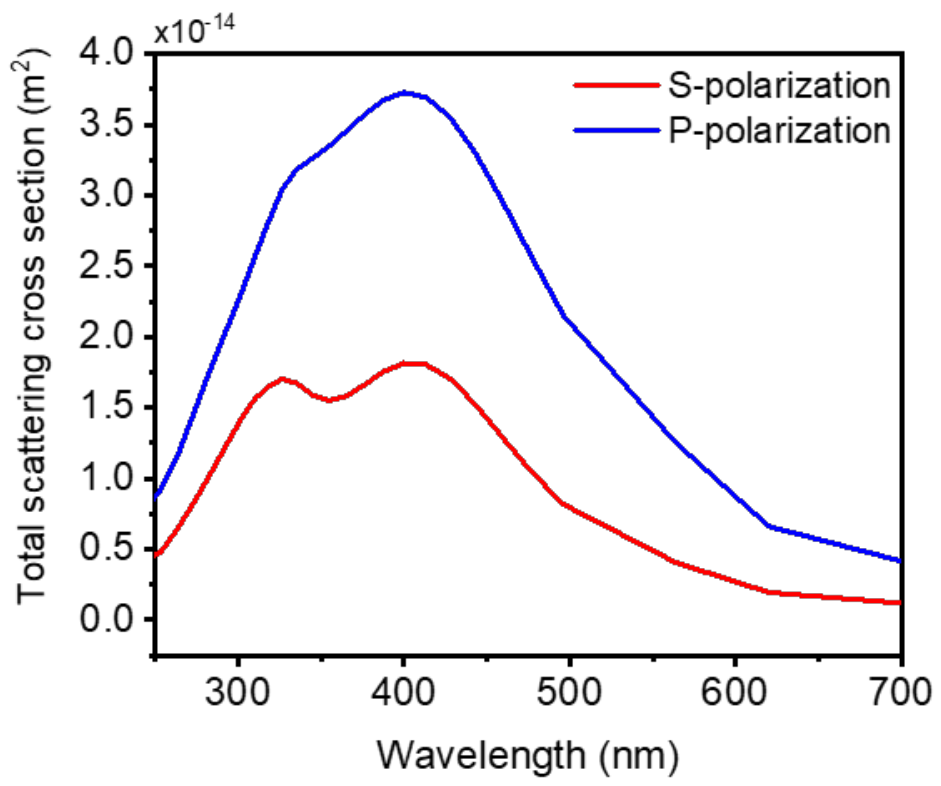

Figure S13. Theoretical total scattering cross section of octopod under the same simulation setup as the dark field scattering spectra simulations in Fig. 1. 


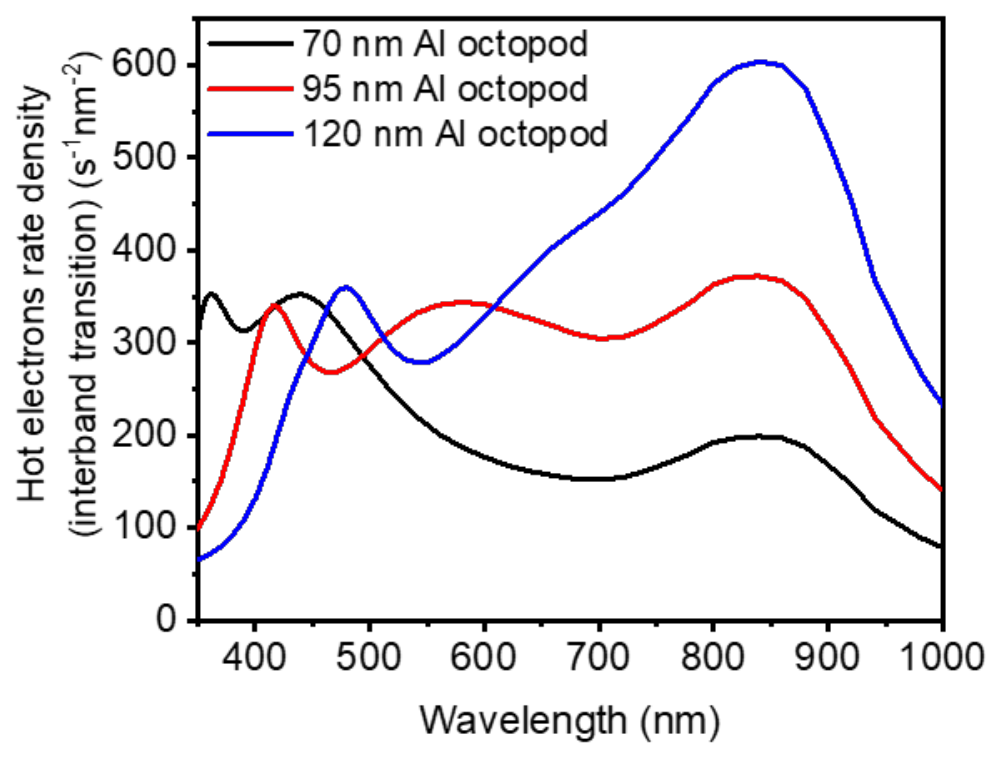

Figure S14. Size effect on Al octopod hot electrons generation rate per surface area via interband transitions under incident light intensity of $1 \mathrm{~W} / \mathrm{cm}^{2}$ and adsorbate resonance linewidth $0.1 \mathrm{eV}$.

\section{References:}

1. Zhang, C.; Zhao, H.; Zhou, L.; Schlather, A. E.; Dong, L.; McClain, M. J.; Swearer, D. F.; Nordlander, P.; Halas, N. J., Al-Pd Nanodisk Heterodimers as Antenna-Reactor Photocatalysts. Nano Lett. 2016, 16, 6677-6682.

2. $\quad$ Rakić, A. D.; Djurišić, A. B.; Elazar, J. M.; Majewski, M. L., Optical Properties of Metallic Films for Vertical-Cavity Optoelectronic Devices. Appl. Opt. 1998, 37, 5271-5283.

3. Palik, E. D., Handbook of Optical Constants of Solids; Academic Press; San Diego, CA, 1985. 